\title{
Simultaneous Design and Controllability Optimization for the Reaction Zone for Furfural Bioproduction
}

Ana Gabriela Romero-García, ${ }^{a}$ Oscar Andrés Prado-Rúbio, ${ }^{\mathrm{b}}$ Gabriel Contreras-Zarazúa,

César Ramírez-Márquez, ${ }^{\mathrm{a}}$ Jorge Humberto Ramírez-Prado. ${ }^{\mathrm{c}}$ Juan Gabriel Segovia-

$$
\text { Hernández, }{ }^{\mathrm{a}^{*}}
$$

${ }^{a}$ Chemical Engineering Department, University of Guanajuato, Campus Guanajuato, Gto., México, 36005.

${ }^{b}$ Departamento de Ingeniería Química, Universidad Nacional de Colombia, Km 9 vía al Aeropuerto La Nubia, Campus La Nubia, Bloque L, Manizales, Caldas, Colombia.

${ }^{c}$ Centro de Investigación Científica de Yucatán, A.C., Unidad de Biotecnología, Calle 43 No. 130, Colonia Chuburná de Hidalgo, CP 97200, Mérida, Yucatán, México 


\begin{tabular}{|l|c|}
\hline \multicolumn{1}{|c|}{ Content } & Page No \\
\hline - Values of El99 impact categories for reactors. & S-2 \\
\hline - Parameters for DETL method. & S-2 \\
\hline - Kinetic parameters obtained from the adjustment of parameters. & S-3 \\
\hline $\begin{array}{l}\text { - Representation of experimental data indicating the reliability for the } \\
\text { proposed model. }\end{array}$ & S-4 \\
\hline - Sensitivity to the parameters identified for the simplified model. & S-5 \\
\hline - Contribution of utilities and equipment costs to TAC & S-5 \\
\hline - Contribution of impact categories to El99 & S-6 \\
\hline
\end{tabular}

Table S1: Values of EI99 impact categories for reactors.

\begin{tabular}{lll}
\hline Impact category & Steel $\left(\right.$ points/kg) $\times \mathbf{1 0}^{-3}$ & Steam $($ points/kg) \\
\hline Carcinogenic & $6.32 \times 10^{-3}$ & $1.04 \times 10^{-4}$ \\
Climate change & $1.31 \times 10^{-2}$ & $1.27 \times 10^{-3}$ \\
Ionizing radiation & $4.51 \times 10^{-4}$ & $1.91 \times 10^{-6}$ \\
Ozone depletion & $4.55 \times 10^{-6}$ & $7.78 \times 10^{-7}$ \\
Respiratory effects & $8.01 \times 10^{-2}$ & $1.56 \times 10^{-3}$ \\
Acidification & $2.71 \times 10^{-3}$ & $1.21 \times 10^{-4}$ \\
Ecotoxicity & $7.45 \times 10^{-2}$ & $2.85 \times 10^{-4}$ \\
Land occupation & $3.730 \times 10^{-3}$ & $8.60 \times 10^{-5}$ \\
Fossil fuels & $5.930 \times 10^{-2}$ & $1.24 \times 10^{-2}$ \\
Mineral extraction & $7.420 \times 10^{-2}$ & $8.87 \times 10^{-6}$ \\
\hline
\end{tabular}

Table S2. Parameters for DETL method.

\begin{tabular}{|c|c|c|c|c|c|}
\hline $\begin{array}{l}\text { Population } \\
\text { size }\end{array}$ & $\begin{array}{l}\text { Number of } \\
\text { generations }\end{array}$ & $\begin{array}{l}\text { Mutation } \\
\text { Factor }(\mathrm{F})\end{array}$ & $\begin{array}{l}\text { Crossover } \\
\text { factor }(\mathrm{Cr})\end{array}$ & $\begin{array}{l}\text { Taboo } \\
\text { List size }\end{array}$ & $\begin{array}{l}\text { Radio } \\
\text { Taboo }\end{array}$ \\
\hline 120 & 834 & 0.6 & 0.8 & 60 & $2.5 \times 10^{-6}$ \\
\hline
\end{tabular}


Table S3. Kinetic parameters obtained from the adjustment of parameters.

\begin{tabular}{|c|c|c|c|c|}
\hline Parameters & Symbol & Units & Value & $\begin{array}{c}\text { Confidence } \\
\text { interval }\end{array}$ \\
\hline $\begin{array}{l}\text { Pre-exponential } \\
\text { constant }\end{array}$ & $\mathrm{ko}_{1}$ & $\min ^{-1}$ & $6.24 \times 10^{8}$ & $\pm 2.28 \times 10^{9}$ \\
\hline Tunable parameter & $b_{1}$ & --- & 1.20 & \pm 0.21 \\
\hline Activation Energy & $\mathrm{E}_{1}$ & $\mathrm{~kJ} / \mathrm{mol}$ & 93.44 & \pm 11.16 \\
\hline $\begin{array}{l}\text { Pre-exponential } \\
\text { constant }\end{array}$ & $\mathrm{ko}_{2}$ & $\min ^{-1}$ & 1.74 & \pm 7.33 \\
\hline Tunable parameter & $b_{2}$ & ---- & 2.06 & \pm 0.56 \\
\hline Activation Energy & $E_{2}$ & $\mathrm{~kJ} / \mathrm{mol}$ & 33.63 & \pm 11.72 \\
\hline \multicolumn{5}{|c|}{ Statistical indicators of parameter adjustment } \\
\hline \multicolumn{3}{|c|}{ Determination Coeficient $\mathrm{r}^{2}$} & \multicolumn{2}{|c|}{0.97} \\
\hline \multicolumn{3}{|c|}{ Adjusted Determination Coeficient $r^{2}$ adj } & \multicolumn{2}{|c|}{0.96} \\
\hline \multicolumn{3}{|c|}{ Parameter Identificability fraction } & \multicolumn{2}{|c|}{$29.83 \%$} \\
\hline
\end{tabular}



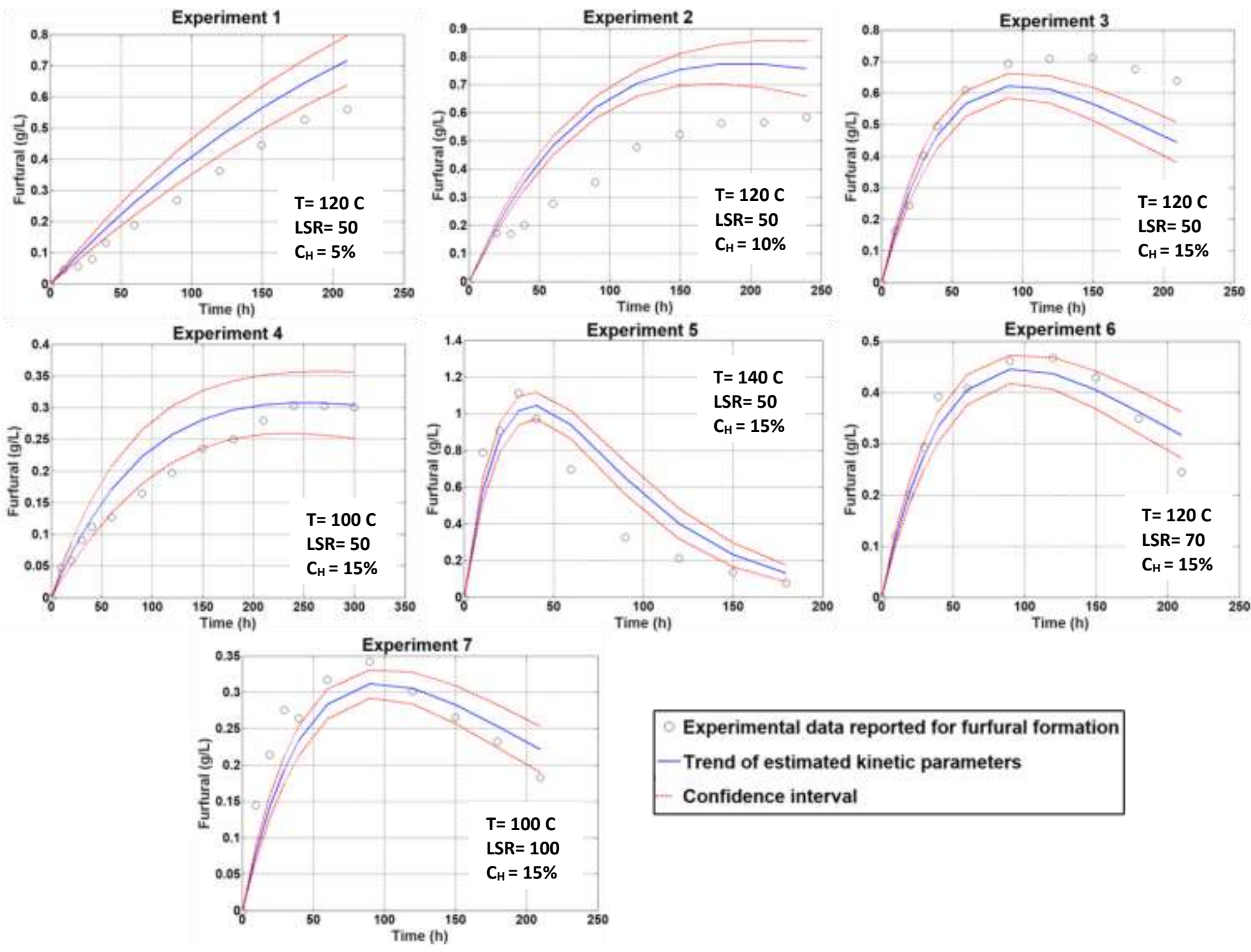

Figure S1: Representation of experimental data indicating the reliability for

the proposed model. 


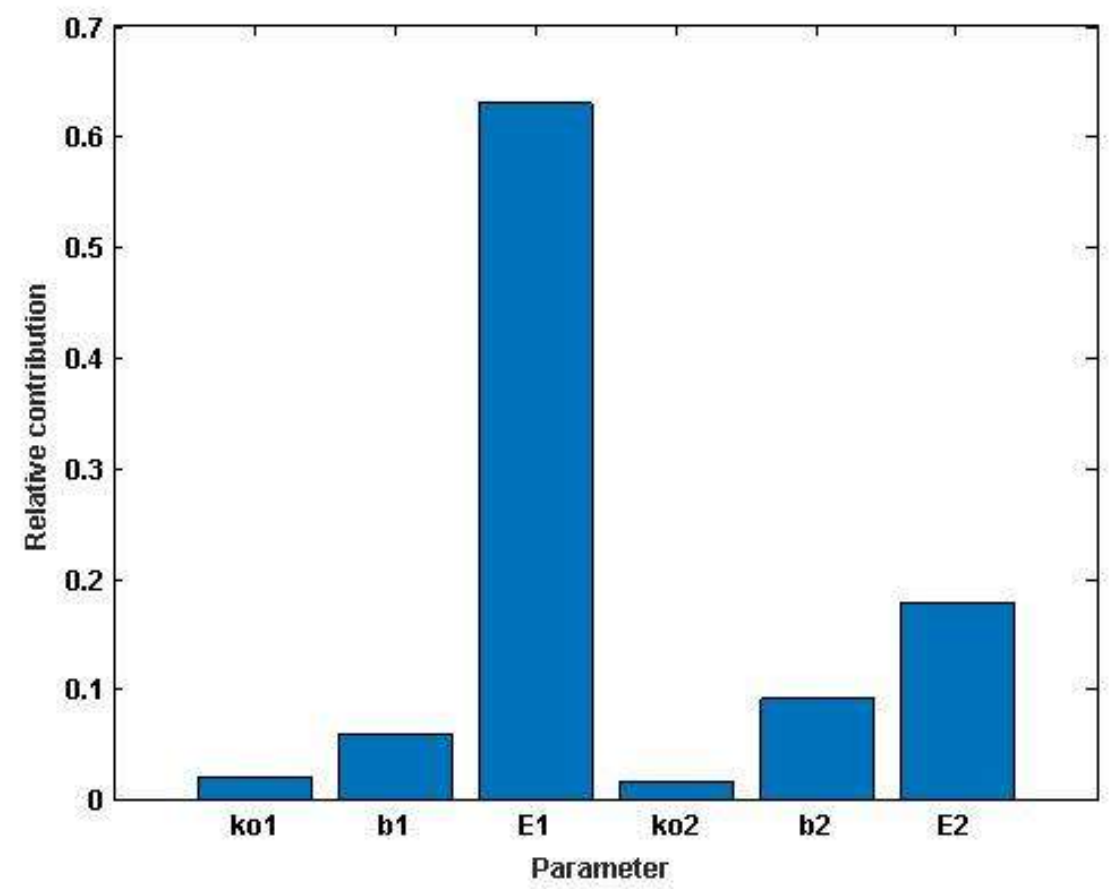

Figure S2: Sensitivity to the parameters identified for the simplified model.

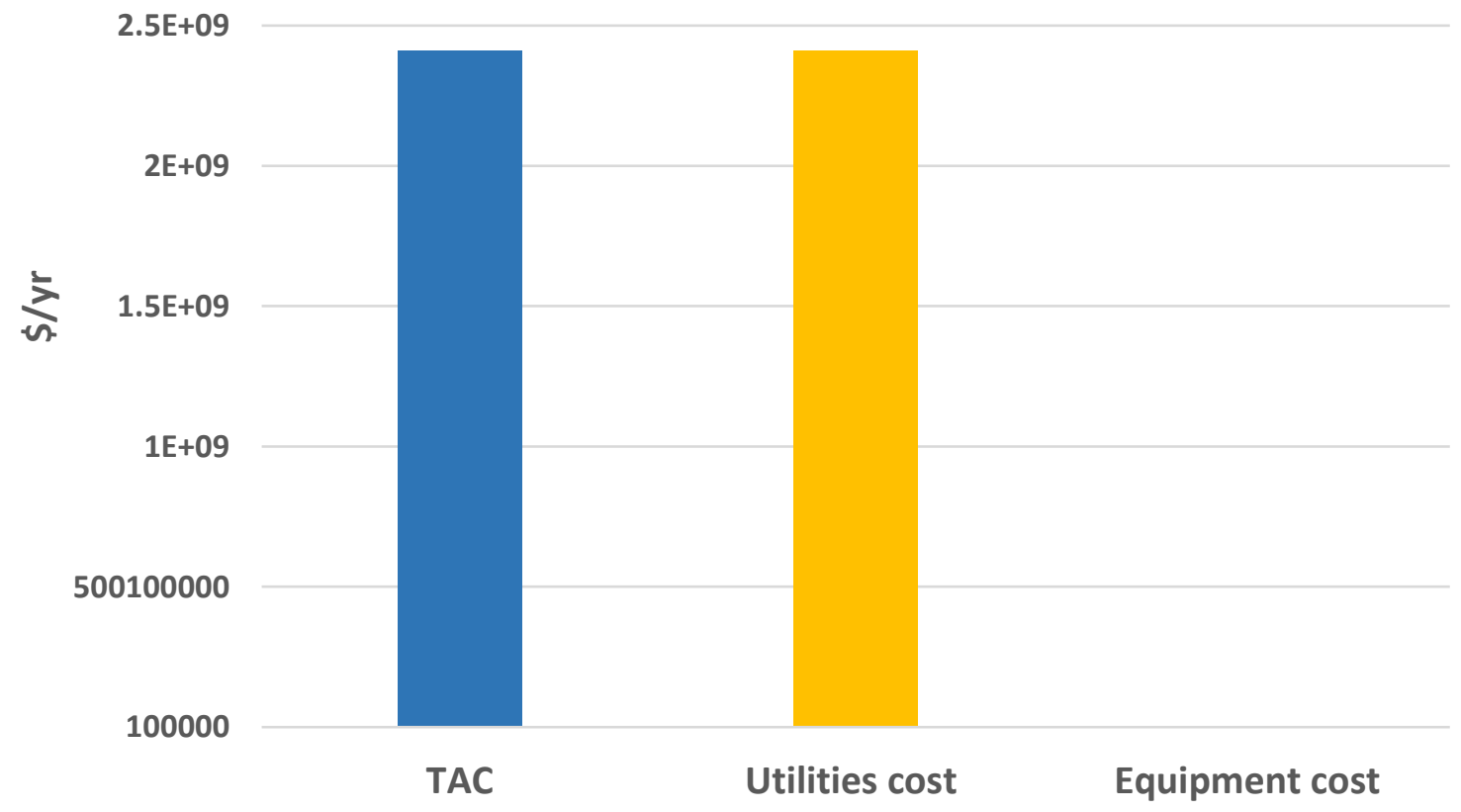

Figure S3: Contribution of utilities and equipment costs to TAC 


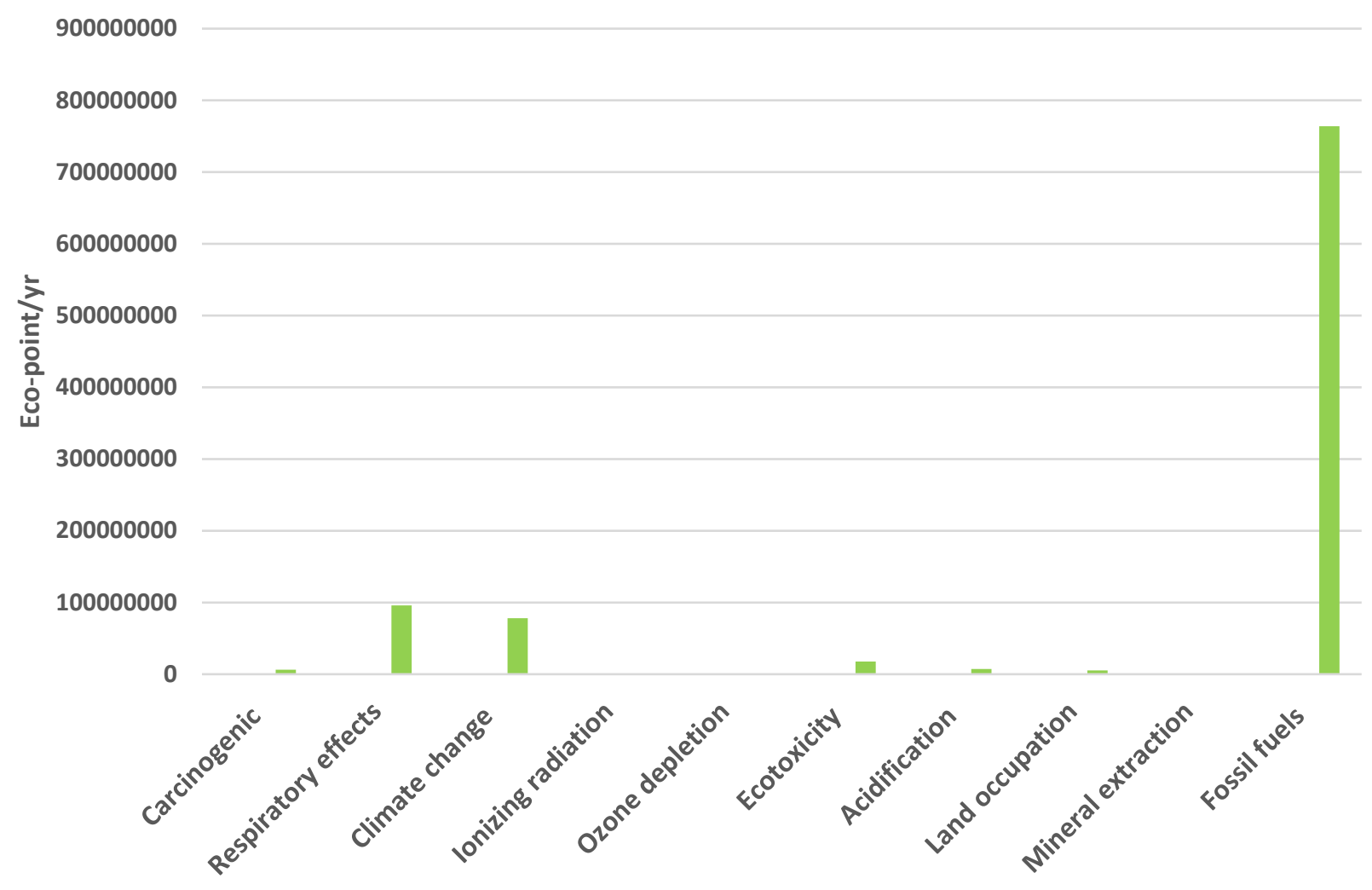

Figure S4: Contribution of impact categories to EI99 